\title{
Niveles de fertilización en dos variedades de maíz morado (Zea mayz L.) en la localidad de Canaán-Ayacucho
}

Levels of fertilization in two varieties of purple corn (Zea mays L.) in the locality of Canaan Ayacucho

Rember Pinedo Taco ${ }^{1}$, Gilberto Rodriguez Soto ${ }^{1}$ y Norma Valverde Reyes ${ }^{1}$

\section{RESUMEN}

Determina la interacción de dos variedades de maíz morado (PMV-581 e INIA615 Negro Canaán) con cuatro niveles de fertilización (NPK) en el rendimiento y contenido de antocianina del cultivo de maíz morado en la Estación Experimental Agraria (EEA) del INIA, en Canaán-Ayacucho. En un Diseño de Bloque Completo al Azar con un arreglo factorial 2V x 4F y cuatro repeticiones, se evaluaron variables biométricas agronómicas, y contenido de antocianina. El mayor rendimiento de mazorca se alcanzó con la variedad INIA-615-Negro Canaán con 3.67 t.ha ${ }^{-1}$ seguida de la variedad PMV-581 con 2.78 t.ha ${ }^{-1}$. Con el nivel de fertilización $\mathrm{f}_{3}$ (120-90-60) se logró el mayor rendimiento de mazorcas (3.69 t.ha ${ }^{-1}$, seguido por los niveles $\mathrm{f}_{4}(120-120-100)$ y $\mathrm{f}_{2}(120-110-80)$ que resultaron estadísticamente iguales. Asimismo, con el nivel de fertilización $\mathrm{f}_{2}(120-110-80)$ se obtuvo el mayor contenido de antocianina en equivalentes de cianidina-3-glucósido $\mathrm{mg} / 100 \mathrm{~g} 2.21$ estadísticamente igual a los niveles de fertilización $\mathrm{f}_{4}(120$ 120-100) 1.64 y f $_{3}(120-90-60) 1.62$. En el contenido de antocianina en equivalentes de cianidina-3-glucósido mg/100g, la variedad Negro Canaán con 1.82 y la variedad PMV-581 con 1.67 son iguales estadísticamente.

Palabras clave: maíz morado; nivel de fertilización; arreglo factorial; contenido de antocianina.

\section{ABSTRACT}

Determines the interaction of two varieties of purple corn (PMV-581 and INIA615 Negro Canaan) with four levels of fertilization in the yield and anthocyanin content of the cultivation of purple corn in the Agricultural Experimental Station (ASE), INIA Canaan-Ayacucho. In a design randomized complete block with a factorial arrangement $2 \mathrm{~V} \times 4 \mathrm{~F}$ and four replications, biometric agronomic

1 Universidad Nacional Agraria La Molina. Lima, Perú. 
variables and anthocyanin content were evaluated. The biggest ear yield was reached with the variety INIA-615-Negro Canaan with 3.67 t.ha ${ }^{-1}$ followed by the variety PMV-581 with 2.78 t.ha $\mathrm{a}^{-1}$. With the level of fertilization $\mathrm{f}_{3}(120-90$ 60) the highest yield of pods ( 3.69 t.ha $\mathrm{r}^{-1}$ ), was achieved, followed by the levels $\mathrm{f}_{4}$ $(120-120-100)$ and $f_{2}(120-110-80)$ that were statistically equal. Also, with the $f_{2}$ fertilization level (120-110-80) the greater content of anthocyanin in equivalents of cyanidin-3-glucoside $\mathrm{mg} / 100 \mathrm{~g} 2.21$ statistically equal to $\mathrm{f}_{4}$ fertilizer levels was obtained (120-120 -100) 1.64 and $f_{3}(120-90-60) 1.62$ In the content of anthocyanin in cyanidin equivalents-3-glucoside $\mathrm{mg} / 100 \mathrm{~g}$, the variety Negro Canaan with 1.82 and PMV-581 range with 1.67 are statistically equal.

Keywords: purple corn; fertilization level; factorial arrangement; anthocyanin content.

\section{INTRODUCCIÓN}

El maíz morado es muy remoto, se cultiva en el Perú desde épocas precolombinas y las diversas variedades provienen de la raza Kculli (Manrique, 1999). Con respecto a las variedades mejoradas, la variedad INIA-615 Negro Canaán fue obtenida por selección recurrente de medios hermanos de una colección realizada por el INIA en las zonas productoras de maíz morado en Ayacucho (Requis, 2012) y la variedad PMV-581 fue obtenida por la Universidad Nacional Agraria La Molina (UNALM) a partir de la variedad Morado de Caraz (Manrique, 1997). El maíz morado se adapta a las condiciones de sierra media entre los 1,800 a $2800 \mathrm{msnm}$, con temperaturas medias anuales de $12^{\circ}$ a $20^{\circ} \mathrm{C}$ y con una precipitación media anual de 500 a $1000 \mathrm{~mm}$ (Manrique, 1997). Pese a su enorme potencial de las 240000 ha de maíz amiláceo sembrado en el país, solo 5000 ha corresponden al maíz morado, siendo las zonas de mayor área de cultivo Arequipa, Ica, Lima, Huánuco, Cajamarca y Ayacucho.

Con respecto a los requerimientos nutricionales y uso de fertilizantes los productores no realizan análisis de suelos previos a la siembra y lo hacen en forma empírica (Requis, 2012). Aunque la planta de maíz usa 16 elementos diferentes, solo tres son necesarios en cantidades relativamente grandes: el nitrógeno $(\mathrm{N})$, el fósforo (P) y el potasio (K). El N es el motor del crecimiento de la planta, es absorbido del suelo bajo forma de nitrato (NO3-) o de amonio (NH4+) (FAO, 2012). El P se clasifica como un nutriente primario, los cultivos lo requieren en cantidades relativamente grandes, así por cada $100 \mathrm{~kg}$ de $\mathrm{P}_{2} \mathrm{O}_{5}$ soluble aplicado al suelo, el cultivo absorbe durante su desarrollo y fructificación de 20 a $60 \mathrm{~kg}$ (Villagarcía y Aguirre, 2012). El K activa más de 60 enzimas, por ello juega un papel vital en la síntesis de carbohidratos y proteínas. Asimismo, mejora el régimen hídrico de la planta y aumenta su tolerancia a sequías, heladas y salinidad. (FAO, 2012). Para la zona de estudio el cultivo de maíz morado tiene requerimientos altos de nitrógeno, fósforo, potasio, siendo lo recomendado el nivel 586-220-100 kg.ha-1 de NPK (Risco, 2007). Con respecto al contenido de 
antocianinas, en el maíz morado peruano se han separado e identificado entre 8 y 11 y las encontradas en mayor proporción son cuatro: cianidina 3-glucósido, cianidina 3-(6-malonil glucósido) y peonidina 3-glucósido. (Aoki et al., 2002; Nobuji et al., 1979). Asimismo, el maíz morado presenta antocianinas del tipo, pelargonidina-3-glucósido a nivel de coronta (Quispe, Aroyo y Gorriti, 2011). El estudio se realizó con la finalidad de determinar la interacción de dos variedades de maíz morado (PMV- 581 e INIA-615 Negro Canaán) con cuatro niveles de fertilización (NPK) en el rendimiento y contenido de antocianina.

\section{MATERIALES Y MÉTODOS}

El experimento se llevó a cabo en la Estación Experimental Agraria (EEA) Canaán-Ayacucho, ubicada a 2735 msnm en la provincia de Huamanga, región Ayacucho (Latitud Sur $12^{\circ} 56^{\prime} 22.8^{\prime \prime}$ y Latitud Oeste $74^{\circ} 14^{\prime}$ 51.5”) Durante el estudio, la temperatura varió entre 6 y $28.6{ }^{\circ} \mathrm{C}$. La humedad relativa entre $74.1 \mathrm{y}$ 84.4 por ciento con una precipitación acumulada de $542.5 \mathrm{~mm}$ (Datos Estación Hidrometeorológica de EEA Canaán-Ayacucho).

Para el estudio se evaluaron las variedades de maíz morado, INIA-615 Negro Canaán y PMV- 581 y cuatro niveles de fertilización (tabla 1). Las fuentes de fueron: Urea (46\% de N), Fostato Diamónico (18 de $\mathrm{N}$ y $46 \% \mathrm{P}_{2} \mathrm{O}_{5}$ ) y Cloruro de potasio $\left(60 \%\right.$ de $\left.\mathrm{K}_{2} \mathrm{O}\right)$. Los tratamientos en estudio fueron ocho, en un Diseño de Bloque Completo al Azar (DBCA) bajo un arreglo factorial 2V x 4F y cuatro repeticiones.

Tabla 1. Variedades y niveles de fertilización utilizados en el experimento

\begin{tabular}{cccccc}
\hline \multicolumn{3}{c}{ Variedades de maíz morado } & \multicolumn{4}{c}{ Niveles de fertilización } \\
Clave & Variedades & Clave & $\mathrm{N}$ & $\mathrm{P}_{2} \mathrm{O}_{5}$ & $\mathrm{~K}_{2} \mathrm{O}$ \\
\hline \multirow{2}{*}{$\mathrm{v}_{1}$} & \multirow{2}{*}{ PMV-581 } & $\mathrm{f}_{1}$ & 18 & 46 & 30 \\
& & $\mathrm{f}_{2}$ & 120 & 90 & 60 \\
\multirow{2}{*}{$\mathrm{v}_{2}$} & \multirow{2}{*}{ INIA - 615 Negro Canaán } & $\mathrm{f}_{3}$ & 120 & 110 & 80 \\
& & $\mathrm{f}_{4}$ & 120 & 120 & 100 \\
\hline
\end{tabular}

La fase experimental duró 180 días a partir de la siembra. Se evaluaron seis variables biométricas: días a la floración (masculina y femenina); altura (de planta y mazorca en $\mathrm{cm}$ ); largo de mazorca, diámetro de mazorca $(\mathrm{cm})$; mientras que las variables agronómicas fueron cinco: número de granos por mazorca, número de hileras por mazorca, peso de mazorca ( $\mathrm{t}$ ), grano y tusa, y rendimiento de mazorcas $\left(\mathrm{t} \mathrm{ha}^{-1}\right) \mathrm{y}$, finalmente se determinó el contenido de antocianina en equivalentes de cianidina-3-glucósido $\mathrm{mg} / 100 \mathrm{~g}$ de muestra. 
La evaluación de variables biométricas se realizó en base al manual gráfico para la descripción varietal de maíz (Carballo, 2010), la lista de descriptores de maíz (IBPGR, 1991) y los descriptores mínimos varietales del INIA (Estrada et al., 2006). Para cada variable en estudio se efectuaron mediciones a 10 plantas tomadas en los dos surcos centrales de cada tratamiento. Para estimar el rendimiento en peso de mazorcas por hectárea en cada unidad experimental se procedió a pesar todas las mazorcas cosechadas. Para la determinación de la intensidad de color de la coronta, se procedió a molerlas y se determinó la absorbancia de la muestra de corontas de maíz morado diluida con solución tampón $\mathrm{C}(\mathrm{a})$ de pH 1.0 y una sustancia tampón C (b) de pH 4.5 tanto a 520 y $700 \mathrm{~nm}$. La muestra diluida en porciones de ensayo se tomó lecturas comparadas con una celda en blanco llena de agua destilada. Luego se procedió a medir la absorbancia dentro de 20 a 50 minutos de la preparación.

\section{RESULTADOS Y DISCUSIÓN}

En el análisis de varianza, tabla 2, no se encontró diferencias significativas estadísticas para bloques variedades, nivel de fertilización e interacción variedad por nivel de fertilización para las variables biométricas. La no significancia de la interacción indica que cada factor fue independiente.

Tabla 2. Análisis de varianza de las variables biométricas

\begin{tabular}{lcccccc}
\hline $\begin{array}{l}\text { Fuentes de } \\
\text { variación }\end{array}$ & $\begin{array}{c}\text { Días a la } \\
\text { floración } \\
\text { masculina }\end{array}$ & $\begin{array}{c}\text { Días a la } \\
\text { floración } \\
\text { femenina }\end{array}$ & $\begin{array}{c}\text { Altura de } \\
\text { planta }\end{array}$ & $\begin{array}{c}\text { Altura de } \\
\text { mazorca }\end{array}$ & $\begin{array}{c}\text { Longitud } \\
\text { de } \\
\text { mazorca }\end{array}$ & $\begin{array}{c}\text { Diámetro } \\
\text { de } \\
\text { mazorca }\end{array}$ \\
\hline Bloque & 1.710 & 0.170 & 0.010 & 0.005 & 0.710 & 0.150 \\
Variedades (V) & 2.000 & 1.130 & 0.010 & 0.002 & 0.430 & 0.360 \\
Fertilización (F) & 0.130 & 1.080 & 0.010 & 0.005 & 1.090 & 0.050 \\
VF & 0.330 & 2.380 & 0.050 & 0.011 & 1.670 & 0.080 \\
Error & 1.400 & 0.830 & 0.020 & 0.007 & 1.160 & 0.140 \\
CV (\%) & 1.310 & 0.930 & 7.330 & 6.120 & 7.980 & 9.160 \\
Promedio & 90.560 & 97.870 & 2.050 & 1.330 & 13.250 & 4.110 \\
\hline
\end{tabular}

*significación de 0,05 de probabilidad, ** significación de 0,01 de probabilidad

La floración masculina y femenina en la variedad PMV-581 se presentó entre los 90.31 y 98.06 días después de la siembra (dds), y en la variedad INIA-615 Negro Canaán entre los 90.81 y 97.69 días respectivamente y, no se halló significación estadística entre los niveles de fertilización para dichas variables (tabla 3). El mayor promedio de altura de planta y mazorca se halló con la $\mathrm{v}_{1} 2.06$ y $1.33 \mathrm{~m}$ seguido por la $\mathrm{v}_{2} 2.04 \mathrm{~m}$ y $1.32 \mathrm{~m}$ respectivamente. 
Tabla 3. Prueba de Comparación de medias Duncan al 0,05 de variedades en promedio de nivel de fertilización para días a la floración, altura de planta y mazorca

\begin{tabular}{lcccc}
\hline Variedad & $\begin{array}{c}\text { Días a la flo- } \\
\text { ración mas- } \\
\text { culina }\end{array}$ & $\begin{array}{c}\text { Días a la floración } \\
\text { femenina }\end{array}$ & Altura de planta & Altura de mazorca \\
\hline $\mathrm{v}_{1}$ & $90.31 \mathrm{a}$ & $98.06 \mathrm{a}$ & $2.06 \mathrm{a}$ & $1.33 \mathrm{a}$ \\
$\mathrm{v}_{2}$ & $90.81 \mathrm{a}$ & $97.69 \mathrm{a}$ & $2.04 \mathrm{a}$ & $1.32 \mathrm{a}$ \\
\hline
\end{tabular}

Los valores con letras iguales no son estadísticamente diferentes

En la tabla 4, con los niveles $\mathrm{f}_{1}, \mathrm{f}_{3} \mathrm{y}_{4}$ la floración masculina ocurrió en promedio a los 90.50 dds. La floración femenina entre los 97.50 y 98.38 dds con los cuatro niveles aplicados. En el maíz morado para las condiciones de Ayacucho, Fernández (2009), afirma que la floración masculina ocurre entre los 82.7 y 86.7 y la floración femenina entre los 96.3 y 98 dds. Para las variables Altura de planta y mazorca, no se hallaron diferencias significativas entre variedades y niveles de fertilización. La mayor altura de mazorca resultó con el nivel $\mathrm{f}_{1}(1.36$ $\mathrm{m})$; asimismo, el mayor promedio de altura de planta $2.09 \mathrm{~m}$ con el nivel $\mathrm{f}_{1}$ y el menor promedio de altura $2.01 \mathrm{~m}$ con el nivel $\mathrm{f}_{4}$. Paucarima (2007), halló $2.84 \mathrm{~m}$, Cruzado (2008), $1.98 \mathrm{~m}$ y $1.20 \mathrm{~m}$ de altura de planta y de mazorca, con el nivel 180-120-60. Resultados aproximados a lo hallado en el trabajo de investigación obtuvieron Pinto (2002) y Huamán (2007) con 1.39 y 1.37 m respectivamente.

Tabla 4. Prueba de Comparación de medias Duncan para nivel de fertilización en promedio de variedades para días a la floración; altura de planta y mazorca

\begin{tabular}{ccccc}
\hline $\begin{array}{c}\text { Nivel de } \\
\text { fertilización }\end{array}$ & $\begin{array}{c}\text { Días a la Floración } \\
\text { masculina }\end{array}$ & $\begin{array}{c}\text { Días a la floración } \\
\text { femenina }\end{array}$ & $\begin{array}{c}\text { Altura de } \\
\text { planta }\end{array}$ & $\begin{array}{c}\text { Altura de } \\
\text { mazorca }\end{array}$ \\
\hline f2 & $90.75 \mathrm{a}$ & $98.38 \mathrm{a}$ & $2.05 \mathrm{a}$ & $1.32 \mathrm{a}$ \\
f1 & $90.50 \mathrm{a}$ & $97.75 \mathrm{a}$ & $2.09 \mathrm{a}$ & $1.36 \mathrm{a}$ \\
f3 & $90.50 \mathrm{a}$ & $97.50 \mathrm{a}$ & $2.05 \mathrm{a}$ & $1.31 \mathrm{a}$ \\
f4 & $90.50 \mathrm{a}$ & $97.88 \mathrm{a}$ & $2.01 \mathrm{a}$ & $1.31 \mathrm{a}$ \\
\hline
\end{tabular}

Los valores con letras iguales no son estadísticamente diferentes

Para las variables longitud y diámetro de mazorcas (tabla 5), en el análisis de varianza no se encontró diferencias significativas para bloques variedades, fertilización e interacción variedad fertilización. La variedad INIA-615 Negro Canaán presentó mayores promedios con respecto a la variedad PMV-581. En cuanto al efecto de los niveles de fertilización para longitud y diámetro de mazorca. El mayor promedio de longitud de mazorca $13.81 \mathrm{~cm}$ y $4.20 \mathrm{~cm}$ con el nivel $\mathrm{f}_{3} \mathrm{y}$, el menor promedio 12,99 y 4,01 $\mathrm{cm}$ con el nivel $\mathrm{f}_{2}$. Paucarima (2007), reporta 
13.58 y $13.15 \mathrm{~cm}$; Roca (1992), $14.9 \mathrm{~cm}$ para un ecotipo local de maíz morado; Huamán (2001), $11.92 \mathrm{~cm}$. Para la variable diámetro de mazorca en el experimento el mayor promedio también resultó $4.20 \mathrm{~cm}$ con $\mathrm{f}_{3}$; para la misma variable Poma (2007), reportó 4.6 cm; Cruzado (2008), 4.82 cm; Pinto (2002), 4.55. Mondalgo (2002), 5.15 y $4.92 \mathrm{~cm}$ respectivamente. Los resultados obtenidos en el experimento para ambas variedades son inferiores a los obtenidos por los autores antes mencionados.

Tabla 5. Comparación de medias Duncan al 0,05 de probabilidades

\begin{tabular}{|c|c|c|c|c|c|}
\hline \multicolumn{3}{|c|}{$\begin{array}{c}\text { Variedades en promedio de niveles de } \\
\text { fertilización }\end{array}$} & \multicolumn{3}{|c|}{$\begin{array}{l}\text { Niveles de fertilización en promedio de varie- } \\
\text { dades }\end{array}$} \\
\hline Variedad & $\begin{array}{l}\text { Largo de } \\
\text { mazorca }\end{array}$ & $\begin{array}{l}\text { Diámetro de } \\
\text { mazorca }\end{array}$ & $\begin{array}{c}\text { Nivel de } \\
\text { fertilización }\end{array}$ & $\begin{array}{l}\text { Longitud de } \\
\text { mazorca }\end{array}$ & $\begin{array}{l}\text { Diámetro de } \\
\text { mazorca }\end{array}$ \\
\hline $\mathrm{v}_{2}$ & $13,64 \mathrm{a}$ & $4,21 \mathrm{a}$ & $\begin{array}{l}\mathrm{f}_{3} \\
\mathrm{f}_{4}\end{array}$ & $\begin{array}{l}13.81 \mathrm{a} \\
13.71 \mathrm{a}\end{array}$ & $\begin{array}{l}4,20 \mathrm{a} \\
4,14 \mathrm{a}\end{array}$ \\
\hline $\mathrm{v}_{1}$ & $13,41 \mathrm{a}$ & $4,00 \mathrm{a}$ & $\begin{array}{l}\mathrm{f}_{1} \\
\mathrm{f}_{2}\end{array}$ & $\begin{array}{l}13.58 \mathrm{a} \\
12.99 \mathrm{a}\end{array}$ & $\begin{array}{l}4,08 \mathrm{a} \\
4,01 \mathrm{a}\end{array}$ \\
\hline
\end{tabular}

Los valores con letras iguales no son estadísticamente diferentes

En el análisis de varianza (tabla 6), para número de granos por hilera e hileras por mazorca que no se encontraron diferencias significativas entre bloque, fertilizantes y la interacción variedad fertilización. Sin embargo, en el factor variedades se encontraron diferencias significativas para las variables peso de mazorca y granos.

Tabla 6. Análisis de varianza variables agronómicas

\begin{tabular}{|c|c|c|c|c|c|}
\hline \multirow[b]{2}{*}{$\begin{array}{l}\text { Fuente de Varia- } \\
\text { ción }\end{array}$} & \multicolumn{5}{|c|}{ Cuadrados Medios } \\
\hline & $\begin{array}{c}\mathrm{N}^{\circ} \text { de } \\
\text { granos } \\
\text { por Hilera }\end{array}$ & $\begin{array}{c}\mathrm{N}^{\mathrm{o}} \mathrm{de} \\
\text { Hileras por } \\
\text { mazorca }\end{array}$ & $\begin{array}{l}\text { Peso de } \\
\text { mazorca }\end{array}$ & $\begin{array}{l}\text { Peso de } \\
\text { granos }\end{array}$ & $\begin{array}{l}\text { Peso de } \\
\text { tusa }\end{array}$ \\
\hline Bloque & 2.77 & 0.32 & 206.75 & 148.78 & 4.37 \\
\hline Variedades (V) & 2.03 & 0.91 & $2926.13^{*}$ & $2363.28^{*}$ & 30.03 \\
\hline Fertilización (F) & 4.41 & 0.14 & 281.58 & 221.36 & 10.45 \\
\hline VF & 1.06 & 0.95 & 246.71 & 166.36 & 22.20 \\
\hline Error & 6.59 & 0.44 & 344.58 & 282.09 & 11.22 \\
\hline CV $(\%)$ & 11.95 & 6.45 & 20.48 & 22.59 & 19.81 \\
\hline Promedio & 21.49 & 10.28 & 90.63 & 74.34 & 16.91 \\
\hline
\end{tabular}

*significación de 0,05 de probabilidad, ** significación de 0,01 de probabilidad 
En la tabla 7, la variedad INIA-615 Negro Canaán resultó con el mayor número de granos por hilera y número de hileras por mazorca (21.74 granos y 10.45 hileras) superando en ambas variables a la variedad PMV-581. Estos resultados son mayores a 10.10 hileras/mazorca hallados por Cruzado (2008). Con el nivel $\mathrm{f}_{3}$ resultó 22.16 como el mayor promedio de granos por hilera, y el menor promedio 20.44 con el nivel $f_{2}$. Para la variable hileras/mazorca, el mayor promedio frente a los demás tratamientos fue 10.48 con $\mathrm{f}_{1}$ mientras que el menor promedio con respecto a los demás tratamientos fue 10.18 con el nivel $\mathrm{f}_{4}$. Fernández (2009) menciona 26.40 como el mayor y 21,28 como el menor promedio de granos por hilera; mientras que Enciso (2005) 24.7 y 25.7 respectivamente.

Tabla 7. Comparación de medias Duncan al 0,05 de probabilidades

\begin{tabular}{|c|c|c|c|c|c|}
\hline \multicolumn{3}{|c|}{$\begin{array}{l}\text { variedades en promedio de niveles de } \\
\text { fertilización }\end{array}$} & \multicolumn{3}{|c|}{ Niveles de fertilización en promedio de variedades } \\
\hline Variedad & $\begin{array}{l}\text { Largo de } \\
\text { mazorca }\end{array}$ & $\begin{array}{l}\text { Diámetro de } \\
\text { mazorca }\end{array}$ & $\begin{array}{c}\text { Nivel de } \\
\text { fertilización }\end{array}$ & $\begin{array}{c}\text { Longitud de } \\
\text { mazorca }\end{array}$ & $\begin{array}{c}\text { Diámetro de } \\
\text { mazorca }\end{array}$ \\
\hline \multirow[t]{2}{*}{$\mathrm{v}_{2}$} & \multirow{2}{*}{$21.74 \mathrm{a}$} & \multirow{2}{*}{$10.45 \mathrm{a}$} & $\mathrm{f}_{3}$ & $22.16 \mathrm{a}$ & $10.23 \mathrm{a}$ \\
\hline & & & $\mathrm{f}_{4}$ & $21.81 \mathrm{a}$ & $10.18 \mathrm{a}$ \\
\hline \multirow[t]{2}{*}{$\mathrm{v}_{1}$} & \multirow{2}{*}{$21.23 \mathrm{a}$} & \multirow{2}{*}{$10.11 \mathrm{a}$} & $\mathrm{f}_{1}$ & $21.53 \mathrm{a}$ & $10.48 \mathrm{a}$ \\
\hline & & & $\mathrm{f}_{2}$ & $20.44 \mathrm{a}$ & $10.25 \mathrm{a}$ \\
\hline
\end{tabular}

Los valores con letras iguales no son estadísticamente diferentes

En la comparación de medias entre variedades no se encontraron diferencias para las variables peso de grano y tusa; pero se halló significación para peso de mazorca (tabla 8). El mayor promedio de peso de mazorca, grano y tusa resultaron con la variedad INIA-615 Negro Canaán con 100.19g, 17.88g y 82.31g respectivamente; y el menor promedio para las mismas variables fueron 81.06, 15.94, y $65.12 \mathrm{~g}$ con la variedad PMV-581. Con respecto a las variables en estudio Mayorga (2011), indica pesos promedio de 84.32, 82.50 y 77,60 g, comparando con los resultados obtenidos en el experimento la variedad $\mathrm{v}_{2}$, resultó mayor en peso promedio de mazorca.

El mejor peso promedio de mazorca fue $95.63 \mathrm{~g}$ con el nivel $\mathrm{f}_{1}$ y el menor $82.25 \mathrm{~g}$ con $\mathrm{f}_{2}$. Para la variable peso de grano el mayor promedio se halló $78.38 \mathrm{~g}$ con el nivel $\mathrm{f}_{3}$ y el menor promedio $67.13 \mathrm{~g} \operatorname{con} \mathrm{f}_{2}$. En peso de tusa el mejor promedio alcanzado $18 \mathrm{~g}$ con el nivel $\mathrm{f}_{1} \mathrm{y}$, el menor promedio con el nivel $\mathrm{f}_{2}$ con $15.38 \mathrm{~g}$. Mayorga (2011), al evaluar el efecto de la fertilización nitrogenada y densidad de siembra en el peso promedio de la variedad PMV-581, reporta pesos promedio 
de 84.32 , 82.50 y 77.60 g respectivamente. En el experimento la variedad INIA615 Negro Canaán resultó mayor con 100.19 g de peso frente a lo reportado por Mayorga (2011); mientras, que con la variedad PMV 581 los resultados en promedio se aproximan. De los resultados obtenidos se puede inferir que para el peso de mazorca, grano y tusa los niveles más altos de fertilización $\left(f_{2}, f_{3} y\right.$ $\mathrm{f}_{4}$ ) no tuvieron efectos significativos frente al nivel $\mathrm{f}_{1}$ en el incremento de peso, posiblemente porque los fertilizantes aplicados no pudieron ser absorbidos por la planta por la característica del suelo ligeramente alcalina $(7.25 \mathrm{pH})$ y por la falta de agua (ausencia de lluvias) en la fase reproductiva de llenado de granos.

Tabla 8. Comparación de medias Duncan al 0,05 de probabilidades

\begin{tabular}{cccccccc}
\hline \multicolumn{2}{c}{$\begin{array}{c}\text { Variedades en promedio de niveles de } \\
\text { fertilización }\end{array}$} & \multicolumn{3}{c}{ Niveles de fertilización en promedio de } \\
variedades
\end{tabular}

En el análisis de varianza para rendimiento de mazorca y contenido de antocianina (tabla 13), se halló significancia para variedades y niveles de fertilización.

Con la variedad INIA-615 Negro se halló un rendimiento de 3.67 t.ha h $^{-1}$, y con la variedad PMV-581 2.78 t.ha-1 (tabla 9). Se puede inferir que la diferencia es por el comportamiento intrínseco varietal. La variedad PMV-581 se adapta y expresa muy bien su potencial de rendimiento hasta los $2600 \mathrm{msnm}$, el experimento fue realizado a $2730 \mathrm{msnm}$. Con relación al contenido de antocianinas no se halló significancia entre las variedades, siendo ambos estadísticamente iguales. Con mayor valor en el contenido de antocianina resultó la variedad Negro Canaán con 1.82 frente a 1,69 de la variedad PMV-581. 
Tabla 9. Análisis de varianza para rendimiento de mazorca y contenido de antocianina y Comparación de medias Duncan al 0,05 de variedades en promedio de fertilización

\begin{tabular}{|c|c|c|c|c|c|}
\hline \multicolumn{3}{|c|}{ Análisis de varianza } & \multicolumn{3}{|c|}{ Comparación de medias Duncan al 0,05 } \\
\hline $\begin{array}{l}\text { Fuente de } \\
\text { variación }\end{array}$ & $\begin{array}{c}\text { Rendimiento } \\
\text { t.ha- } \mathrm{ha}^{-1}\end{array}$ & $\begin{array}{l}\text { Contenido } \\
\text { antocianina }\end{array}$ & Variedades & Rendimiento & $\begin{array}{c}\text { Contenido de } \\
\text { antocianina }\end{array}$ \\
\hline Bloques & 0.39 & 0.17 & & & \\
\hline Variedades (V) & $6.38^{*}$ & 0.07 & & 367 & 182 \\
\hline Fertilización (F) & 1.18 & $0.38^{*}$ & $v_{2}$ & $J, 0 / \mathrm{d}$ & $1,02 \mathrm{a}$ \\
\hline VF & 0.62 & 0.07 & & & \\
\hline Error & 0.52 & 0.05 & & & \\
\hline CV $(\%)$ & 22.26 & 12.99 & $\mathrm{v}_{1}$ & $2,78 \mathrm{~b}$ & $1,69 \mathrm{a}$ \\
\hline Promedio & 3.23 & 1.75 & & & \\
\hline
\end{tabular}

* Significación de 0,05 de probabilidad, ** significación de 0,01 de probabilidad

Los valores con letras iguales no son estadísticamente diferentes.

El mayor rendimiento obtenido 3.69 t.ha ${ }^{-1}$ con el nivel de fertilización $f_{3}$, seguido por los niveles de fertilización $\mathrm{f}_{4} \mathrm{y}_{2}$ siendo iguales estadísticamente los tres niveles, y el rendimiento más bajo 2.78 t.ha ${ }^{-1}$ con el nivel de fertilización $\mathrm{f}_{1}$, estadísticamente igual a los niveles de fertilización $\mathrm{f}_{4}$ y $\mathrm{f}_{2}$. Solano (1999), con el nivel 120-180-120 halló 4.96 t.ha ${ }^{-1}$ como el mayor rendimiento; Paucarima (2007), 10.08 y Fernández (2009) 8.70 t.ha . $^{-1}$ En el experimento los rendimientos alcanzados fueron menores a los reportados por los tres autores antes citados. Risco (2007), menciona que la fase de floración es el periodo más crítico porque de ella va a depender el cuajado y la cantidad de producción obtenida. Para contenido de antocianinas (tabla 10) se encontró diferencias significativas entre los niveles de fertilización. El mayor valor de contenido de antocianina en equivalentes de cianidina-3-glucósido $\mathrm{mg} / 100 \mathrm{~g}$ de muestra fue 2.21 con el nivel de fertilización $\mathrm{f}_{2}$, seguido por los niveles $\mathrm{f}_{4}$ y $\mathrm{f}_{3}$ y que resultaron estadísticamente iguales, y el menor contenido hallado 1.55 con el nivel $\mathrm{f}_{1}$, que resultó estadísticamente diferente al nivel $f_{2}$, y similar a los niveles $f_{4}, \mathrm{y}_{3}$.

Tabla 10. Comparación de medias Duncan al 0.05 de nivel de fertilización en promedio de variedades para rendimiento de mazorca $\left(\mathrm{t} \mathrm{ha}^{-1}\right)$ y contenido de antocianina

\begin{tabular}{cccc}
\hline $\begin{array}{c}\text { Nivel de } \\
\text { Fertilización }\end{array}$ & Rendimiento & $\begin{array}{c}\text { Nivel de } \\
\text { Fertilización }\end{array}$ & Contenido de antocianina \\
\hline $\mathrm{f}_{3}$ & $3.69 \mathrm{a}$ & $\mathrm{f}_{2}$ & $2.21 \mathrm{a}$ \\
$\mathrm{f}_{4}$ & $3.35 \mathrm{a} \mathrm{b}$ & $\mathrm{f}_{4}$ & $1.64 \mathrm{a} \mathrm{b}$ \\
$\mathrm{f}_{2}$ & $3.09 \mathrm{a} \mathrm{b}$ & $\mathrm{f}_{3}$ & $1.62 \mathrm{a} \mathrm{b}$ \\
$\mathrm{f}_{1}$ & $2.78 \mathrm{~b}$ & $\mathrm{f}_{1}$ & $1.55 \mathrm{~b}$ \\
\hline
\end{tabular}


Justiniano (2010), puntualiza como el mayor valor 59,25 de intensidad de color value. Fernández (2009), menciona que el índice de tinción del maíz morado bajo condiciones de la EEA Canaán Ayacucho, varía de 4.11 hasta 4.69. Mientras que Huamán (2007), asevera el valor 4.45 de índice de tinción en la variedad INIA Negro Canaán.

\section{CONCLUSIONES}

El mayor rendimiento de mazorca se alcanzó con la variedad INIA-615-Negro Canaán con 3.67 t.ha ${ }^{-1}$. Con el nivel de fertilización $\mathrm{f}_{3}$ (120-110-80) se encontró el mayor rendimiento de mazorcas; sin embargo, aun siendo mayor en cuanto a NPK con el nivel f3 se logró el menor resultado y, estadísticamente igual a los niveles de fertilización $\mathrm{f}_{4}(120-120-100) 3,35$ t.ha $^{-1} \mathrm{y} \mathrm{f}_{2}(120-90-60) 3.09$ t.ha ${ }^{-1}$. En el contenido de antocianina en equivalentes de cianidina-3-glucósido $\mathrm{mg} / 100 \mathrm{~g}$, la variedad Negro Canaán y la variedad PMV-581 resultaron estadísticamente iguales. Con el nivel de fertilización $\mathrm{f}_{2}$ (120-110-80) se halló el mayor contenido de antocianina en equivalentes de cianidina-3-glucósido en $\mathrm{mg} / 100 \mathrm{~g}$, mientras que con y el nivel $f_{1}$ se halló el menor contenido.

\section{REFERENCIAS BIBLIOGRÁFICAS}

Aoki, Hiromitsu; N, kuze, Noriko; kato, Yoshiaki. 2002. Anthocyanins isolated from purple corn (Zea mays L.). Foods \& food Ingred. Vol. 8. N 199 Japan. 41-45.

Carballo, Aquiles. 2010. Manual gráfico para la descripción varietal en maír. (Zea mays L.). Colegio de post graduados en ciencias agrícolas. 2da edición. Chihuahua: SAGARPA.

Cruzado, Luís. 2008. Efecto de fertilización fosforo - potásica en el cultivo de maíz morado (Zea mays 1). Tesis para optar el título de Ing. Agr. Lima, Perú. UNALM. $87 \mathrm{p}$.

Enciso, Luís. 2005. Infuencia de la densidad de plantas en el rendimiento de dos variedades de maíz morado (Zea mays 1.) y frijol reventón (Phaseolus vulgaris l.) Canaán a 2760 msnm. Tesis para optar el título de Ing. Agrónomo. Ayacucho: UNSCH.

Estrada, Rigoberto; Medina, Tulio; Roldan, Agripina. 2006. Manual para caracterización in situ de cultivos nativos. Conceptos y procedimientos. Lima: INIA. 167.

FAO (Organización de las Naciones Unidas para la Alimentación y la Agricultura, IT). 2012. Los fertilizantes y su uso. Segunda edición. Roma, Italia. 77. 
Fernández, Honorato. 2009. Aplicación de roca fosfórica y diatomita incubada en microorganismos en el cultivo de maíz morado (Zea mays L.) en la Estación Experimental del INIA Canaán Ayacucho. Tesis para optar el título de Ing. Agr. Ayacucho. Universidad Nacional San Cristóbal de Huamanga. Ayacucho: UNSCH. 140.

Huamán, Edwin. 2007. Influencia del guano de isla en el rendimiento de dos variedades de maí morado (Zea mays L) Canaán a 2750 msnm. Tesis para optar el título de Ingeniero Agrónomo. Universidad Nacional San Cristóbal de Huamanga. Ayacucho: UNSCH.

Huamán, Oscar. 2001. Estudio de la asociación de maí morado (Zea mays L.) con tres lineas de frijol (Phaseolus vulgaris L.) en dos momentos de siembra en Canaán Ayacucho. Tesis para optar el título de Ingeniero Agrónomo. Universidad Nacional San Cristóbal de Huamanga. Ayacucho: UNSCH.

IBPGR (International Board for Plant Genetic Resources). 1991. Descriptors for maize. International Maize and Wheat Improvement Center. Mexico city: International Board for Plant Genetic Resources.

Justiniano, Erasmo. 2010. Fenología e intensidad de color en corontas del maíz morado (Zea mayz. L.) en sus diferentes estados de desarrollo en la localidad de La Molina. Tesis para optar el título de Mg. Sc. Universidad Nacional Agraria La Molina. Lima: EPG. 77.

Manrique, Antonio. 1997. El maíz en el Perú. Lima: Consejo Nacional de Ciencia y Tecnología (CONCYTEC) 362.

Manrique, Antonio. 1999. El maiz morado peruano (Zea mays L. amilaceae st.). Lima: INIA. Boletín No 2-99. 24.

Mayorga, Amalia. 2011. Efecto de la densidad de siembra y de fertilización nitrogenada en el rendimiento de maí morado (Zea mays L.) cv. PMV-581, bajo riego por goteo. Tesis para optar el título de Ingeniero Agrónomo. Universidad Nacional Agraria la Molina: Lima UNALM. 118.

Mondalgo, David. 2002. Comparativo de rendimiento de mair morado (Zea mays L.) con tres fórmulas de fertilización N-P-Ky dos densidades de siembra en la EE A Canaán Ayacucho a 2750 msnm. Tesis para optar el título de Ingeniero Agrónomo. Universidad Nacional San Cristóbal de Huamanga. Ayacucho: UNSCH.

Nakatani, Nobuji; Fukuda, Hilton; Fuwa, Hidetsugu. 1979. Major anthocyanin of Bolivian purple corn (Zea mays L.). Agricultural and Biological Chemistry. Vol. 43. $\mathrm{N}^{\circ}$ 2. 389-391.

Paucarima, Edgar. 2007. Respuesta de maíz morado (Zea mays L.) a cuatro fórmulas de abonamiento y tres densidades de siembra Canaán a 2750 msnm Ayacucho. Tesis para optar el título Ingeniero Agrónomo. Universidad Nacional San Cristóbal de Huamanga. Ayacucho: UNSCH. 
Pinto, Edwin. 2002. Selección mazorca bilera modificada en maíz morado negro (Zea mays L). Tesis para optar el título el título de Ingeniero Agrónomo. Universidad Nacional San Cristóbal de Huamanga. Ayacucho: UNSCH.

Poma, Ivet. 2007. Efecto de la fertilización química y orgánica con y sin la aplicación de microorganismos eficientes (EM) en el rendimiento de maíz, morado (Zea mays l.) $\mathrm{c} v$. $P M V$-581. Tesis para optar el título de Ingeniero Agrónomo. Universidad Nacional Agraria La Molina. Lima: UNALM.

Quispe, Fredy; Arroyo, Karim.; Gorriti, Arrilmí. 2011. «Características morfológicas y químicas de 3 cultivares de maíz morado (Zea mays L.) de Arequipa. Soc Quim Perú. Vol. 77. N 3. 205-217.

Requis, Florencio. 2012. Manejo agronómico del maí morado en los valles interandinos del Perú. Lima: INIA. Boletín No 1-12. 23.

Risco, Martha. 2007. Conociendo la cadena productiva del maíz morado en Ayacucho. SOLID - Perú. 88 p.

Roca, Oscar. 1992. Rendimiento de dos variedades de frijol bajo tres densidades de siembra en asociación con maí morado. Tesis para optar el título de Ingeniero Agrónomo. Universidad Nacional San Cristóbal de Huamanga. Ayacucho: UNSCH

Solano, Ricardo. 1999. Efecto de la fertirrigación de NPK en el rendimiento y el contenido de antocianina de tres variedades de maíz morado (Zea mays L.) bajo r.l.a.f: goteo. Tesis para optar el título de Ing. Agr. Universidad Nacional Agraria La molina. Lima: UNALM.

Villagarcía, Sven y Aguirre, Guillermo. 2012. Manual de uso de fertilizantes. Lima: Universidad Nacional Agraria La Molina. Departamento Académico de Suelos.

Presentado: $10 / 02 / 2017$

Aceptado: 21/04/2017

\section{Correspondencia}

Rember E. Pinedo T.

rpinedo@lamolina.edu.pe 